\title{
CLINICAL RESEARCH ARTICLE Six-monthly palivizumab prophylaxis effectively reduced RSV-associated hospitalization rates of preterm infants in a subtropical area: a population-based cohort study
}

\author{
Yuh-Jyh Lin ${ }^{1}$, Ching-Hu Chung ${ }^{2}$, Hsin $\mathrm{Chi}^{2,3}$ and Chyi-Her Lin ${ }^{1,4}$
}

\begin{abstract}
BACKGROUND: To evaluate the effects of 6-monthly palivizumab on respiratory syncytial virus-associated hospitalization (RSVH) in preterm infants in an area without RSV seasonality.

METHODS: RSV prophylaxis with 6-monthly palivizumab in infants born at gestational age (GA) $\leq 28$ weeks or those born at GA 29-35 weeks with bronchopulmonary dysplasia (BPD) was implemented in Taiwan since 2010. RSVH, use of mechanical ventilation (MV), admission to intensive care unit (ICU), length of hospital stay, and annual mortality were compared between the historical control group (no prophylaxis, 2008-2009) and the prophylaxis group (2011-2013).

RESULTS: The annual RSVH rates decreased in the target population and in subgroups of infants who received prophylaxis (all target infants: odds ratio [OR], 0.43; 95\% confidence interval [CI], 0.29-0.65). No difference was observed in MV and ICU usage and 1-year mortality in the $\leq 28$ weeks subgroup. In the GA 29-35 weeks with BPD subgroup, ICU usage and 1-year mortality rates were significantly reduced with palivizumab prophylaxis regimen. A significant decrease was noted in the annual mortality and ICU admission rates of infants who received prophylactic treatment.

CONCLUSION: Six-monthly palivizumab treatment reduced the RSVH rate, ICU usage, and annual mortality rates of target infants in an area without RSV seasonality.
\end{abstract}

Pediatric Research (2019) 86:628-634; https://doi.org/10.1038/s41390-019-0492-7

\section{INTRODUCTION}

Respiratory syncytial virus (RSV) is the most common cause of lower respiratory tract infections (LRTIs) in children and a leading cause of hospitalization in infants worldwide. ${ }^{1}$ RSV infection has been linked to recurrent wheezing during childhood and pediatric asthma, and is an important cause of mortality in the early months of life in the developing world. ${ }^{2}$ Studies have estimated that $1-4 \%$ of infants require admission to hospital for RSV-associated LRTI before 1 year of age. ${ }^{3}$

Both prematurity and bronchopulmonary dysplasia (BPD) are significant risk factors for severe RSV infection. Infected infants with these two factors tend to have a higher risk of hospitalization, longer hospital stays, and require intensive care and ventilatory support compared with infected infants without these risk factors.

Currently, the only approved preventive treatment for severe RSV diseases is a neutralizing anti-RSV humanized monoclonal antibody (palivizumab) for high-risk populations, including preterm infants and preterm infants with BPD. The American Academy of Pediatrics (AAP) first published guidance for palivizumab prophylaxis among these high-risk infants to prevent their hospitalization during RSV seasons in 1998 and subsequently updated this guidance in 2003, 2009 , and 2014 according to new available evidence. ${ }^{4-7}$ These revisions narrowed the population range recommended for prophylaxis with an emphasis on the reduced risk and severity of
RSV diseases. Social and demographic factors, such as population density, air pollution, public health policy, latitudes, climates, and viral-specific factors of RSV, might influence epidemics at national and regional levels. ${ }^{8-14}$ Whether this guidance could be applied to areas with different epidemiologic features is not well known. A single-hospital study suggested that the AAP recommendation for RSV prophylaxis may not be applicable in Taiwan, where there is no RSV seasonality. ${ }^{15}$ Furthermore, the annual incidence of RSVassociated hospitalization (RSVH) was the highest among infants under 6 months of age. ${ }^{16}$ Based on these data and AAP guidance published before 2014, a regimen of 6-monthly palivizumab prophylaxis beginning 3-5 days before the date of first discharge was proposed for high-risk preterm infants. ${ }^{15}$

Starting in December 2010, this RSV prophylaxis regimen has been implemented in Taiwan for infants born at gestational age (GA) $\leq 28$ complete weeks or for those born at 29-35 complete weeks with BPD, and the regimen proved to be effective in a single-center study. ${ }^{17}$ As RSV surveillance is an important issue worldwide, a comprehensive study of the RSV prophylactic strategy for these infants in subtropical climates is essential for global RSV prevention strategies. We hypothesized that this novel RSV prophylaxis regimen can reduce the incidence of RSVH after first discharge in the high-risk preterm infants. This population-based cohort study, therefore, was to assess the

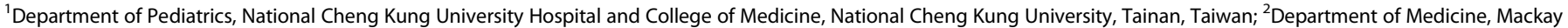

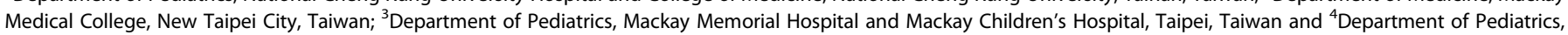
E-Da Hospital and College of Medicine, I-Shou University, Kaohsiung, Taiwan

Correspondence: Chyi-Her Lin (neonate@mail.ncku.edu.tw)

These authors contributed equally: Yuh-Jyh Lin, Ching-Hu Chung

Received: 17 January 2019 Revised: 19 June 2019 Accepted: 20 June 2019

Published online: 1 July 2019 
effects of 6-monthly doses of palivizumab on preventing RSVH in high-risk preterm infants.

\section{MATERIALS AND METHODS}

Data source

A single-payer and compulsory National Health Insurance (NHI) program has been implemented in Taiwan since 1995. The NHI claims that the database includes data regarding complete outpatient visits, hospital admissions, prescriptions, diseases, and vital status of over $99 \%$ of the 23 million population in Taiwan. We established a longitudinal cohort of each beneficiary by linking several computerized administrative and claim datasets. Using the Birth Certificate Application Database, the infants were identified on the basis of the civil identification number unique to each beneficiary and date of birth. All data used in the present study were anonymized and were obtained through a formal application to the Health and Welfare Data Science Center at the Ministry of Health and Welfare, Taiwan (H103161). The study protocol was approved by the Joint Institutional Review Board, Department of Health, Taiwan (14-S-019).

\section{Study population}

We searched the Birth Certificate Application Database for the source population who were born between 2008 and 2013 to identify all different GA of newborns. GA was estimated according to the first day of the mother's last menstrual period or through obstetrical examination. Infants were divided into two subgroups according to their GA: $\leq 28$ complete weeks and 29-35 complete weeks with BPD. Although the palivizumab prophylaxis regimen was implemented in December 2010 in Taiwan, high-risk infants born between January and December 2010 were provided with palivizumab prophylaxis. Because this may be a confounder (unsystematic recruitment bias/lack of standardization) while interpreting the study results, infants born in the year 2010 were excluded from the analysis. Infants who were born between January 2008 and December 2009, who did not receive palivizumab prophylaxis, were considered as the historical control group. Infants who were born between January 2011 and December 2013 and received monthly palivizumab prophylaxis were classified as the palivizumab prophylaxis group. All the infants' data were retrieved for at least 1 year after their first discharge. BPD was identified on the basis of one of the following discharge diagnoses: International Classification of Diseases, Ninth Revision, Clinical Modification (ICD-9-CM) codes 7707 (chronic respiratory disease arising in the perinatal period), 51883 (chronic respiratory failure), and 51884 (acute and chronic respiratory failure). In the analysis, patients were classified as having RSV infection if they had one hospital admission with one of the following discharge diagnoses: ICD-9-CM codes 079.6 (RSV infection), 480.1 (RSV pneumonia), and 466.11 (RSV bronchiolitis).

\section{Outcome measurement}

The primary outcome is the effectiveness of this prophylactic strategy, which was evaluated by the decrease in annual RSVH between the two groups. We generated Kaplan-Meier curves indicating the percentage of the target population that was RSVHfree according to whether they received palivizumab. Furthermore, the historical control and palivizumab subgroups were compared with respect to the mortality rate within 1 year after the date of first discharge. In addition, those with RSVH were evaluated for secondary outcomes, which included the need of mechanical ventilation (MV) and intensive care, the total length of stay (LOS) in the hospital (days), and total hospitalization costs within 1 year after the first discharge. The need for MV was identified using the ICD-9-CM procedure code 96.7x (other continuous invasive MV). To protect personal privacy, Health and Welfare Data Science Center only allows exporting the results with more than two cases in each event. We, therefore, summarized case number as $<3$ if that situation applied. The RSV epidemiology can have "mild" years, with relatively lower rates of RSVH, and "severe" years, with higher rates of RSVH. Since the term infants did not receive palivizumab before and after the implementation of this regimen, we also evaluated the RSVH rate among term infants in both periods to examine whether there was any change in RSVH epidemiology in these two periods.

Data analyses

We used the $x^{2}$ test to compare RSVH incidence, need for MV and intensive care, and annual mortality of infants after their first discharge between the prophylaxis and control groups. Statistical significance in contingency tables with any number $<6$ was tested using Fisher's exact test. Continuous variables were assessed using one-way analysis of variance and the Newman-Keuls multiple comparison test. The log-rank test was used to compare the Kaplan-Meier curves from the historical control and palivizumab groups. All statistical analyses were performed using SAS version 9.1 (SAS Institute, Cary, NC, USA). A $P$ value $<0.05$ was regarded as statistically significant.

\section{RESULTS}

Study population

We linked the Birth Certificate Application Database and $\mathrm{NH}$ claims datasets through civil identification number. The study cohort included 234,015 infants born between 2008 and 2009 and 377,320 infants born between 2011 and 2013 (Fig. 1).

\section{Demographic data of study population}

Table 1 presents the distributions of sex, GA, and birth weight in these cohorts. Compared with the infants born in the historical control period, those born in the palivizumab prophylaxis period had $^{1}$ lower GA and birth weight in all target populations, ${ }^{2}$ lower birth weight in the GA $\leq 28$ weeks subgroup, ${ }^{3}$ lower proportion of male infants and lower GA and birth weight in the GA 29-35 weeks with BPD subgroup, and ${ }^{4}$ lower proportion of male newborns with underlying comorbidities for high-risk RSV infection.

RSVH of infants with or without palivizumab prophylaxis

Table 2 shows that infants in the target population and all subgroups without palivizumab prophylaxis exhibited associations with higher RSVH rates within 12 months after their first date of discharge from the hospital compared with their counterparts. The prevalence rate of RSVH among term infants (GA $\geq 37$ weeks) in Taiwan was higher in 2011-2013 (palivizumab prophylaxis period) than in 2008-2009 (historical control period). However, the proportion of male infants and the incidence of underlying comorbidities with RSVH risk in these term infants were significantly lower during the palivizumab prophylaxis period (Table 1).

RSVH trends of target infants with or without palivizumab prophylaxis

Kaplan-Meier curves were used to analyze the difference in RSVH rate between infants with and without palivizumab prophylaxis (Fig. 2). In the first 3 months, the RSVH rate among infants without palivizumab prophylaxis was $2.19 \%$ in both the $G A \leq 28(N=14)$ and GA of 29-35 weeks with BPD $(N=4)$ subgroups. By contrast, the RSVH rate among infants with palivizumab prophylaxis was $0.68 \%(N=7)$ in the $G A \leq 28$ weeks subgroup and $0.00 \%(N=0)$ in the GA of 29-35 weeks with BPD subgroup. A total of 9 months after discharge from the hospital, the RSVH rate among infants without palivizumab prophylaxis increased to $5.48 \%(N=35)$ in the GA $\leq 28$ weeks subgroup and $7.10 \%(N=13)$ in the GA of 29-35 weeks with BPD subgroup. The RSVH rate of infants with palivizumab prophylaxis during this period was only $2.42 \%$ 


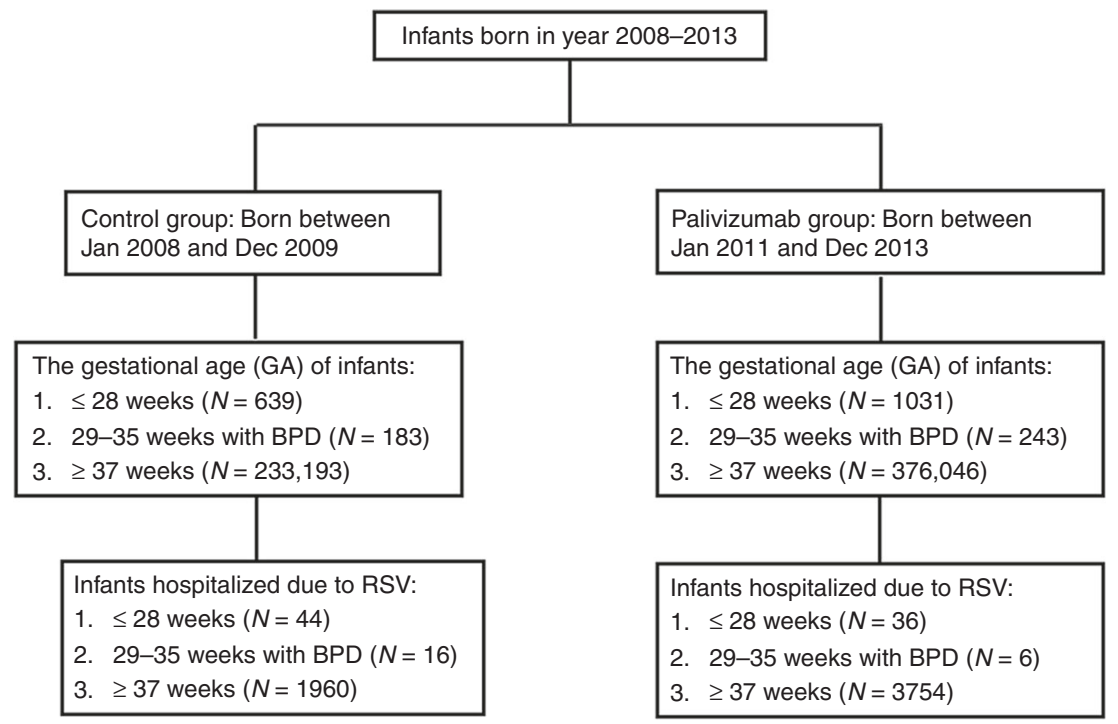

Fig. 1 Flow chart of infants included in the study

$(N=25)$ in the $\mathrm{GA} \leq 28$ weeks subgroup and $1.23 \%(N=3)$ in the GA of 29-35 weeks with BPD subgroup. These results indicated a higher percentage of RSVH-free individuals in both subgroups of the targeted population after receiving palivizumab prophylaxis $(P=0.021$ in the $\mathrm{GA} \leq 28$ weeks subgroup and $p=0.016$ in $\mathrm{GA}$ of 29-35 weeks with the BPD subgroup).

Mortality within 12 months after the date of first discharge with or without palivizumab prophylaxis

For all target infants, the annual all-cause mortality rate after the date of first discharge was lower during the palivizumab prophylaxis period than during the historical control period (Table 2). Moreover, the annual all-cause mortality rate was significantly lower in both subgroups after implementing palivizumab prophylaxis (Table 2). However, for all the target infants and those in the subgroups, the annual respiratory-related mortality rate was not statistically different between the two study periods (Table 2).

Intensive care unit usage, MV usage, LOS, and total hospitalization costs for RSVH treatment with or without palivizumab prophylaxis for infants

Intensive care unit (ICU) usage, MV usage, LOS, and hospitalization costs were used to assess the severity of illness for RSVH. ${ }^{18}$ The proportion of infants admitted to the ICU with RSVH decreased after palivizumab prophylaxis in the target population (Table 3). However, the proportion of patients admitted to the ICU was similar for both periods in the $\mathrm{GA} \leq 28$ weeks subgroup.

The LOS and total medical cost associated with RSVH within the first year after the date of first discharge was not different between the two study periods among the target infants. By contrast, for the infants in the GA of 29-35 weeks with BPD subgroup in the palivizumab prophylaxis period, both LOS and total hospitalization costs of RSVH were significantly lower than those during the historical control period (Table 4).

\section{DISCUSSION}

This population-based cohort study revealed that prophylaxis of 6monthly palivizumab reduced the rate of RSVH by approximately $60 \%$ within 12 months after the date of first discharge from hospitals in all target infants. Additionally, this regimen decreased the rate of RSVH of infants born at GA $\leq 28$ complete weeks and those born at a GA of 29-35 complete weeks with BPD. These results are consistent with the results of the IMpact-RSV study. ${ }^{19}$ The IMpact-RSV study reported that palivizumab prophylaxis resulted in a $55 \%$ reduction in $\mathrm{RSVH}$ rate, $38 \%$ reduction in $\mathrm{RSVH}$ rate of preterm infants with $\mathrm{BPD}$, and $78 \%$ reduction in $\mathrm{RSVH}$ rate of preterm infants without BPD. Furthermore, the relative risk reduction (RRR) of RSVH in preterm infants with GA $\leq 28$ complete weeks without BPD in the IMpact trial was $80.4 \%$, whereas it was $57 \%$ in this study, which also included infants with BPD. ${ }^{20}$ The RRR of RSVH in the IMpact BPD preterm cohort at GA $\leq 35$ complete weeks was $38 \%$, whereas in this study, which focused on infants with GA of 29-35 complete week with BPD only, the RRR was 74\% (1.9-fold higher). ${ }^{20}$ Reduction rates between this study and the IMpact-RSV study differed and may be due to the different targeted population, RSV prophylaxis regimens, and RSV epidemiological regions.

To investigate whether RSVH prevalence rates in Taiwan were similar in the historical control and palivizumab prophylaxis periods, the prevalence rate of RSVH was also evaluated for infants of $G A \geq 37$ weeks. We discovered that the RSVH rate in the palivizumab prophylaxis period was higher than that in the historical control period. In addition, the proportion of female infants and the incidence of underlying comorbidities related to a higher risk of RSV infection in these term infants were lower in the palivizumab prophylaxis period compared with the historical control period. Thus, reduction in the RSVH rate in the target population was more likely due to palivizumab prophylaxis and less likely due to other temporal changes that might have influenced the RSVH.

We also found that this regimen reduced the annual all-cause mortality rate within 12 months after the date of first discharge from the hospital. However, it did not reduce the rate of respiratory-related mortality. The reason for this discrepancy is unknown but may be related to the small sample size of infants with respiratory-related mortality.

In the target population, ICU usage was reduced in infants with RSVH in the palivizumab prophylaxis period, but no difference was observed between infants in the two subgroups in terms of ICU usage. By contrast, MV usage was similar in the two study periods among the target population. These findings indicate that palivizumab prophylaxis for the target population not only reduced RSVH rates but also resulted in a decreased proportion of patients requiring intensive care during RSVH. As described previously, the Health and Welfare Data Science Center of Taiwan only allows the exporting of data with more than two cases in 
Table 1. Demographic data of study population

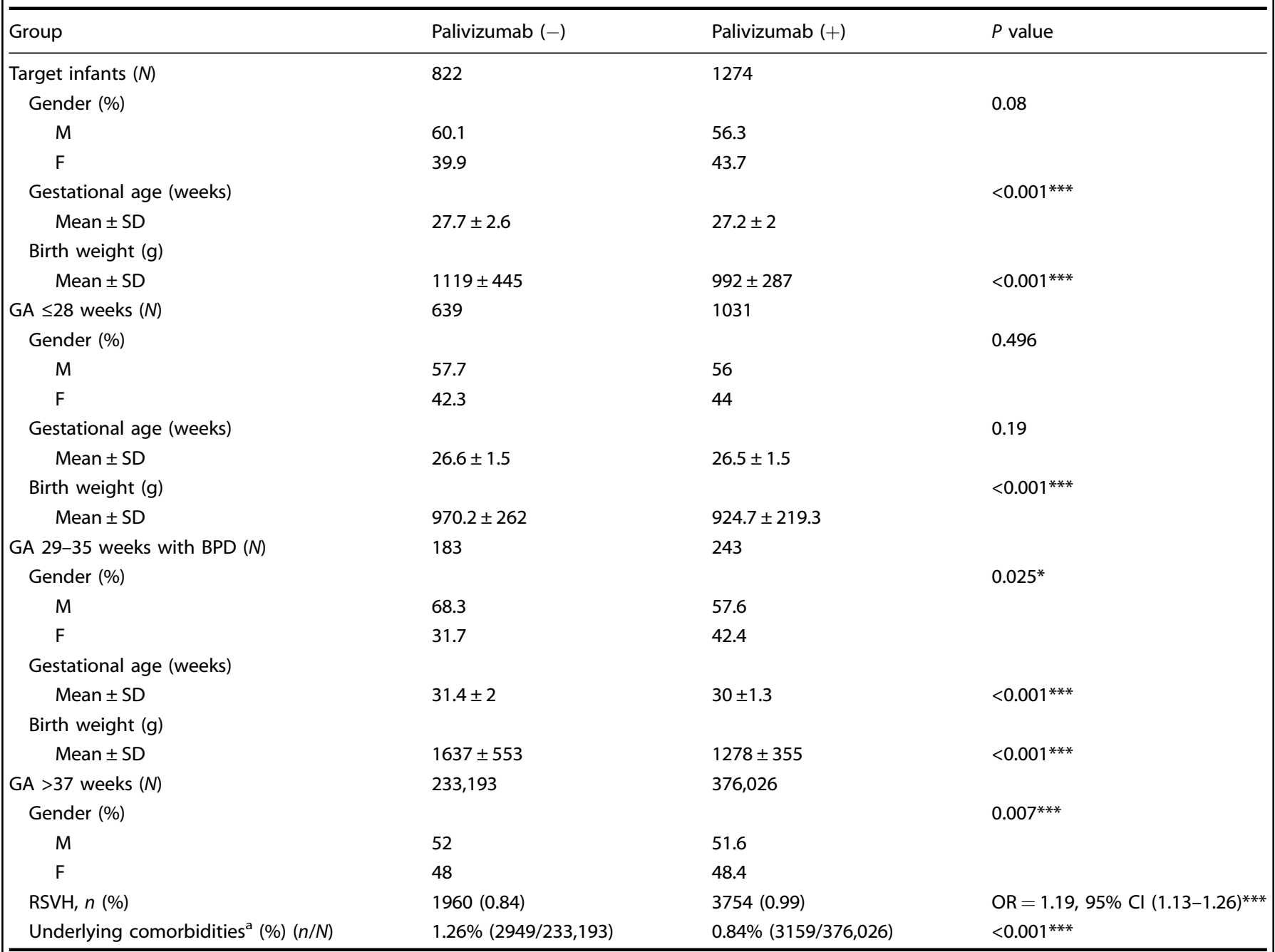

Down syndrome (758.0); diseases of myoneural junction and muscle/neuromuscular disease (358-359); cystic fibrosis (277.0); congenital abnormalities of the airway/congenital anomalies respiratory system (748); immune disorders $(279,288)$

$R S V H$ respiratory syncytial virus-associated hospitalization, $O R$ odds ratio, $C l$ confidence interval, GA gestational age, $B P D$ bronchopulmonary dysplasia, $I C D-9$ International Classification of Diseases, Ninth Revision

${ }^{*} P<0.05,{ }^{* *} P<0.001$, when comparing groups with and without palivizumab prophylaxis

a Underlying comorbidities with high-risk for RSV infection included (ICD-9 coding)

each categorical event. We therefore summarized figures of ICU or MV usage as $<3$ in the results. This rendered the interpretation of data difficult when the case number could be 1 or 2 .

The incidence of RSV-associated LRTI varies widely in different reports. ${ }^{13,21-23}$ One of the factors is the variation in the seasonality of disease burden within and across global regions with different latitudes. ${ }^{23}$ While mid-winter epidemics of RSV infection tend to occur in temperate zones, seasonality is less pronounced and occasionally absent in regions with tropical or arctic climates.

The RSV season begins in the fall and continues into spring in most of the regions in the United States and temperate climate regions such as Canada and northern Europe. However, yearround RSV activity has been reported in Florida (latitude: $21^{\circ} 30^{\prime} \mathrm{N}$ to $31^{\circ} \mathrm{N}$ ) and Puerto Rico (latitude: $18^{\circ} \mathrm{N}$ ). ${ }^{24-27}$ Taiwan (latitude: $23^{\circ}$ $0^{\prime} \mathrm{N}$ to $25^{\circ} 5^{\prime} \mathrm{N}$ ) is located in a subtropical region. Relevant studies have disclosed prolonged seasonality or a year-round pattern of RSV infection with biannual peaks in spring and fall in Taiwan, which was similar to the RSV activity patterns reported in previous studies. ${ }^{15,16,28}$ These RSV infection patterns may be explained by the absence of great temperature changes and precipitations in different seasons in Taiwan. ${ }^{29}$

This study found that the most vulnerable period for severe RSV infection in these high-risk infants was within 9 months after the date of first discharge from the hospital. The Taiwanese guideline for RSV prophylaxis, therefore, recommends 6-monthly doses of palivizumab in the high-risk preterm infants. ${ }^{15,17}$ In the cotton rat model, serum palivizumab concentrations of $25-30 \mu \mathrm{g} / \mathrm{mL}$ during the time of RSV challenge produced a mean $99 \%$ reduction in the pulmonary RSV load and at serum concentrations of $>40 \mu \mathrm{g} / \mathrm{mL}$ produced a minimum of $99 \%$ reduction in pulmonary RSV infection in all animals. ${ }^{30}$ In the IMpact-RSV study, the serum trough concentrations (mean $\pm S D$ ) before the second, third, fourth, and fifth doses were $37 \pm 21,57 \pm 41,68 \pm 51$, and $72 \pm$ $50 \mu \mathrm{g} / \mathrm{mL}$, respectively. ${ }^{19}$ The mean serum trough concentrations increased after repeated doses and demonstrated a wide range and variability. These findings support the rationale for monthly weight-based palivizumab doses throughout the RSV season to avoid RSVH. ${ }^{31}$ We speculate that the mean serum trough concentrations before the sixth dose will be higher than the 
Table 2. Incidence of RSVH and annual mortality in infants with or without palivizumab prophylaxis

\begin{tabular}{lccc}
\hline Outcomes & Palivizumab $(-)$ & Palivizumab $(+)$ & Odds ratio $(95 \%$ Cl) \\
\hline RSVH, $n / N$ (\%) & & & \\
$\quad$ Target population & $60 / 822(7.30)$ & $42 / 1274(3.30)$ & $0.43(0.29-0.65)^{* * *}$ \\
GA $\leq 28$ weeks & $44 / 639(6.89)$ & $36 / 1031(3.49)$ & $0.49(0.31-0.77)^{* *}$ \\
GA 29-35 weeks with BPD & $16 / 183(8.74)$ & $6 / 243(2.46)$ & $0.26(0.1-0.7)^{* *}$ \\
All-cause mortality, $n / N(\%)$ & & & \\
$\quad$ Target population & $33 / 822(4.01)$ & $18 / 1274(1.41)$ & $0.34(0.19-0.61)^{*}$ \\
GA $\leq 28$ weeks & $18 / 639(2.82)$ & $7 / 243(2.88)$ & $0.37(0.17-0.79)^{*}$ \\
GA 29-35 weeks with BPD & $15 / 183(8.20)$ & & $0.33(0.13-0.83)^{*}$ \\
Respiratory-related mortality, $n / N(\%)$ & & $6 / 1274(0.47)$ & \\
Target population & $9 / 822(1.09)$ & $5 / 1031(0.48)$ & $0.42(0.15-1.21)$ \\
GA $\leq 28$ weeks & $6 / 639(0.94)$ & $<3 / 243(0.41)$ & $0.51(0.16-1.69)$ \\
GA 29-35 weeks with BPD & $3 / 183(1.64)$ & $0.25(0.03-2.4)$ \\
\hline
\end{tabular}

Target population included infants born at GA $\leq 28$ weeks and GA 29-35 weeks with BPD

$R S V H$ respiratory syncytial virus-associated hospitalization, $C l$ confidence interval, GA gestational age, $B P D$ bronchopulmonary dysplasia

${ }^{*} P<0.05,{ }^{* * P}<0.01,{ }^{* * *} P<0.001$, when comparing groups with and without palivizumab prophylaxis

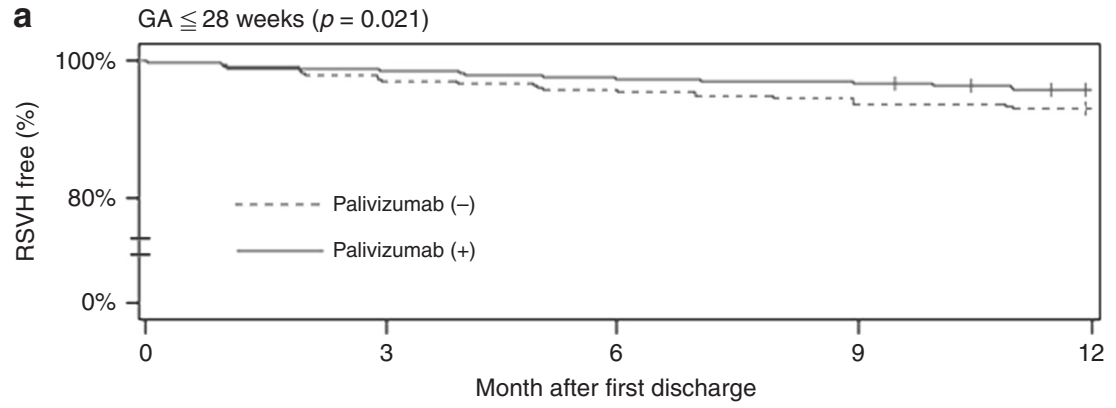

b $\quad$ GA 29-35 weeks/BPD $(p=0.016)$

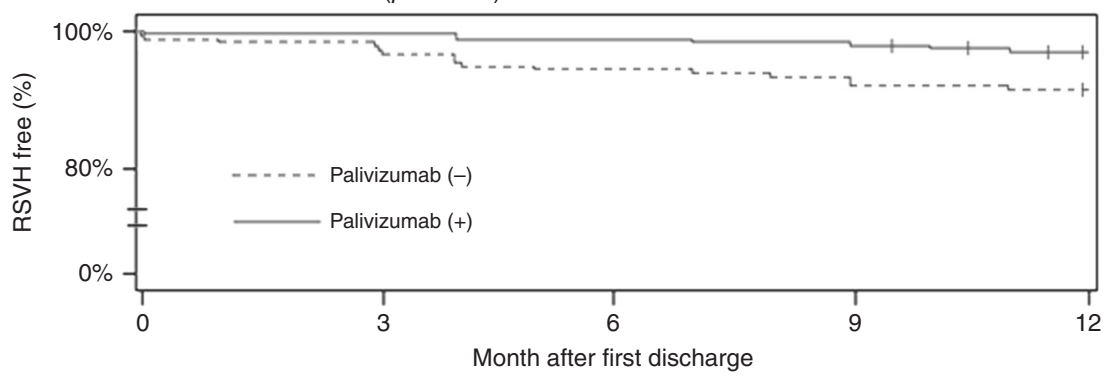

Fig. 2 Kaplan-Meier curves were constructed to illustrate the percentage of the target population that was respiratory syncytial virusassociated hospitalization (RSVH)-free according to whether they received palivizumab. There was a higher percentage of RSVH-free individuals in both subgroups of the targeted population after receiving palivizumab prophylaxis

trough level before the fifth dose, and this would provide protection against RSV infection beyond 7-9 months after the date of first discharge from the hospital; this may constitute a better regimen in a region without RSV seasonality.

We also observed reduced LOS and hospitalization costs of RSVH in the treated infants of GA 29-35 weeks with BPD, when comparing with control subgroups after their first discharge. These findings inferred that there could be a potential less usage of medical resource and reduced costs. Whether the increased medication cost for six doses of palivizumab prophylaxis can be balanced with the potential reduced usage of medical resources warrants further cost-benefit analysis.
The strength of this report is that it was a population-based cohort study based in a population-dense island in a subtropical region and all the high-risk infants in Taiwan are included and covered by the universal NHI program. The infants in this study received 6-monthly palivizumab doses instead of 3-5 monthly doses during RSV seasons, as in other studies.

This study had its limitations. First, RSV bronchiolitis is identified through ICD-9-CM coding of the claim dataset. We are not clear whether these diagnoses are confirmed by what laboratory tests. Recent prospective population-based studies of laboratory-confirmed cases have reported 50\% less RSVH than retrospective studies. ${ }^{7}$ In addition, laboratory tests available for 
Table 3. Usage of ICU or mechanical ventilation during RSVH in groups with or without palivizumab prophylaxis

\begin{tabular}{|c|c|c|c|}
\hline \multicolumn{4}{|l|}{ ICU usage, $n / N$ (\%) } \\
\hline Target population & $15 / 60(25)$ & $<3 / 42(<7.14)$ & $0.23(0.06-0.85)^{*}$ \\
\hline GA $29-35$ weeks with BPD & $11 / 16(68.8)$ & $0 / 6(0)$ & $0.091(0.005-1.64)^{a}$ \\
\hline \multicolumn{4}{|l|}{ Mechanical ventilation, $n / N(\%)$} \\
\hline GA 29-35 weeks with BPD & $7 / 16(43.8)$ & $0 / 6(0)$ & $0.05(0.003-0.904)^{*, a}$ \\
\hline
\end{tabular}

\begin{tabular}{|c|c|c|c|}
\hline \multicolumn{4}{|l|}{ LOS, mean \pm SD (median) } \\
\hline $\mathrm{GA} \leq 28$ weeks & $9.59 \pm 6.99(8.0)$ & $11.93 \pm 16.77(8.0)$ & 0.397 \\
\hline GA 29-35 weeks with BPD & $12.31 \pm 8.19(8.5)$ & $6.86 \pm 2.41(8.0)$ & $<0.001^{* * *}$ \\
\hline $\mathrm{GA} \leq 28$ weeks & $2096 \pm 3326(1107)$ & $3072 \pm 5636(1364)$ & 0.318 \\
\hline GA 29-35 weeks with BPD & $3630 \pm 3501(2167)$ & $1299 \pm 1051$ (929) & $<0.001^{* * * *}$ \\
\hline
\end{tabular}

the diagnosis of RSV infection included the rapid antigen assay, antigen test with cell culture, reverse transcription-polymerase chain reaction assay, and serological tests. All these tests have different sensitivity and specificity in the diagnosis of RSV infection, and RSVH incidence might be different when confirmed through different laboratory tests. ${ }^{32}$ Moreover, the incidence of RSV-associated LRTI was reported to be 6-fold higher when measured through active, compared with passive, surveillance. ${ }^{33}$ Second, due to ethical issues, we can only perform a retrospective cohort study using historical data as control from claimed datasets. Third, in addition to palivizumab prophylaxis, changes in hospitalization criteria, changes in ICD9-CM coding practices, coinfection with other respiratory viruses, or other temporal changes might have influenced comparisons between the prophylactic and control periods. Because term infants did not receive palivizumab in either the control or prophylactic periods, their annual RSVH rate may serve as a surrogate for the factors that did not change between the two periods. Despite increased RSVH among term infants in the palivizumab prophylaxis period, the RSVH rate in the target population was reduced in that period. This indicates that palivizumab prophylaxis effectively reduced RSVH.

In conclusion, this population-based cohort demonstrated that prophylaxis with 6-monthly doses of palivizumab in high-risk infants particularly in the BPD subgroup significantly reduced the rates of RSVH, admission to ICU, and annual all-cause mortality within 12 months after the date of first discharge in an area without RSV seasonality.

\section{ACKNOWLEDGEMENTS}

We thank Dr. William Oh for his critical review and helpful advice on this paper. This study was supported by a grant from Mackay Medical College, Taipei, Taiwan. Grant No. MMC-1051D01.

\section{AUTHOR CONTRIBUTIONS}

Y.-J.L. conceptualized and designed the study, drafted the initial manuscript, and reviewed and revised the manuscript. H.C. conceptualized and designed the study, and reviewed and revised the manuscript. C.-H.C. designed the data collection instruments, collected data, carried out the initial analyses, and reviewed and revised the manuscript. C.-H.L. conceptualized and designed the study, coordinated, supervised data collection, and critically reviewed the manuscript for important intellectual content. All authors approved the final manuscript as submitted and agree to be accountable for all aspects of the work.

\section{ADDITIONAL INFORMATION}

Competing interests: The authors declare no competing interests.

Publisher's note: Springer Nature remains neutral with regard to jurisdictional claims in published maps and institutional affiliations.

\section{REFERENCES}

1. Shi, T. et al. Global, regional, and national disease burden estimates of acute lower respiratory infections due to respiratory syncytial virus in young children in 2015: a systematic review and modelling study. Lancet 390, 946-958 (2017).

2. Polack, F. P. The changing landscape of respiratory syncytial virus. Vaccine 33, 6473-6478 (2015). 
3. Krilov, L. R. et al. Partial palivizumab prophylaxis and increased risk of hospitalization due to respiratory syncytial virus in a Medicaid population: a retrospective cohort analysis. BMC Pediatr. 14, 261 (2014).

4. Pediatrics, A. A. P. Prevention of respiratory syncytial virus infections: indications for the use of palivizumab and update on the use of RSV-IGIV. American Academy of Pediatrics Committee on Infectious Diseases and Committee of Fetus and Newborn. Pediatrics 102, 1211-1216 (1998).

5. American Academy of Pediatrics Committee on Infectious D, Committee on F, Newborn. Revised indications for the use of palivizumab and respiratory syncytial virus immune globulin intravenous for the prevention of respiratory syncytial virus infections. Pediatrics 112, 1442-1446 (2003).

6. American Academy of Pediatrics,. From the American Academy of Pediatrics: policy statements-modified recommendations for use of palivizumab for prevention of respiratory syncytial virus infections. Pediatrics 124, 1694-1701 (2009).

7. American Academy of Pediatrics. Updated guidance for palivizumab prophylaxis among infants and young children at increased risk of hospitalization for respiratory syncytial virus infection. Pediatrics 134, 415-420 (2014).

8. Chen, C. J. et al. Epidemiology of respiratory syncytial virus in children with lower respiratory tract infection. Acta Paediatr. Taiwan 46, 72-76 (2005).

9. Choudhuri, J. A. et al. Effect of altitude on hospitalizations for respiratory syncytial virus infection. Pediatrics 117, 349-356 (2006).

10. Welliver, R. C. Sr. Temperature, humidity, and ultraviolet B radiation predict community respiratory syncytial virus activity. Pediatr. Infect. Dis. J. 26, S29-S35 (2007).

11. Noyola, D. E. \& Mandeville, P. B. Effect of climatological factors on respiratory syncytial virus epidemics. Epidemiol. Infect. 136, 1328-1332 (2008).

12. Meerhoff, T. J., Paget, J. W., Kimpen, J. L. \& Schellevis, F. Variation of respiratory syncytial virus and the relation with meteorological factors in different winter seasons. Pediatr. Infect. Dis. J. 28, 860-866 (2009).

13. Sloan, C., Moore, M. L. \& Hartert, T. Impact of pollution, climate, and sociodemographic factors on spatiotemporal dynamics of seasonal respiratory viruses. Clin. Transl. Sci. 4, 48-54 (2011).

14. Hervas, D., Reina, J. \& Hervas, J. A. Meteorologic conditions and respiratory syncytial virus activity. Pediatr. Infect. Dis. J. 31, e176-e181 (2012).

15. Hsu, C. H. et al. Prolonged seasonality of respiratory syncytial virus infection among preterm infants in a subtropical climate. PLOS ONE 9, e110166 (2014).

16. $\mathrm{Chi}, \mathrm{H}$. et al. Epidemiological study of hospitalization associated with respiratory syncytial virus infection in Taiwanese children between 2004 and 2007. J. Formos. Med. Assoc. 110, 388-396 (2011).

17. Chi, H. et al. A novel six consecutive monthly doses of palivizumab prophylaxis protocol for the prevention of respiratory syncytial virus infection in high-risk preterm infants in Taiwan. PLoS ONE 9, e100981 (2014).

18. Moler, F. W. \& Ohmit, S. E. Severity of illness models for respiratory syncytial virusassociated hospitalization. Am. J. Respir. Crit Care Med 159, 1234-1240 (1999).
19. The IMpact-RSV Study Group. Palivizumab, a humanized respiratory syncytial virus monoclonal antibody, reduces hospitalization from respiratory syncytial virus infection in high-risk infants. The IMpact-RSV Study Group. Pediatrics 102, 531-537 (1998).

20. Notario, G. et al. Respiratory syncytial virus-related hospitalization in premature infants without bronchopulmonary dysplasia: subgroup efficacy analysis of the IMpact-RS V trial by gestational age group. Pediatr. Health Med. Ther. 2014, 6 (2014).

21. Panozzo, C. A., Fowlkes, A. L. \& Anderson, L. J. Variation in timing of respiratory syncytial virus outbreaks: lessons from national surveillance. Pediatr. Infect. Dis. J. 26, S41-S45 (2007).

22. Paes, B. A., Craig, C., Pigott, W. \& Latchman, A. Seasonal respiratory syncytial virus prophylaxis based on predetermined dates versus regional surveillance data. Pediatr. Infect. Dis. J. 32, e360-e364 (2013).

23. Haynes, A. K. et al. Respiratory syncytial virus circulation in seven countries with Global Disease Detection Regional Centers. J. Infect. Dis. 208(Suppl. 3), S246-S254 (2013).

24. Centers for Disease Control and Prevention (CDC). Respiratory syncytial virus activity-United States, July 2008-December 2009. Morb. Mortal. Wkly Rep. 59, 230-233 (2010)

25. Centers for Disease Control and Prevention (CDC). Respiratory syncytial virus activity-United States, July 2011-January 2013. Morb. Mortal. Wkly Rep. 62, 141-144 (2013)

26. Light, M. Respiratory syncytial virus seasonality in southeast Florida: results from three area hospitals caring for children. Pediatr. Infect. Dis. J. 26, S55-S59 (2007).

27. Halstead, D. C. \& Jenkins, S. G. Continuous non-seasonal epidemic of respiratory syncytial virus infection in the southeast United States. South Med. J. 91, 433-436 (1998).

28. Lee, J. T. et al. Epidemiology of respiratory syncytial virus infection in northern Taiwan, 2001-2005-seasonality, clinical characteristics, and disease burden. J. Microbiol. Immunol. Infect. 40, 293-301 (2007).

29. Tsai, H. P., Kuo, P. H., Liu, C. C. \& Wang, J. R. Respiratory viral infections among pediatric inpatients and outpatients in Taiwan from 1997 to 1999. J. Clin. Microbiol. 39, 111-118 (2001).

30. Johnson, S. et al. Development of a humanized monoclonal antibody (MEDI-493) with potent in vitro and in vivo activity against respiratory syncytial virus. J. Infect. Dis. 176, 1215-1224 (1997).

31. Makari, D., Checchia, P. A. \& Devincenzo, J. Rationale for full-season dosing for passive antibody prophylaxis of respiratory syncytial virus. Hum. Vaccin. Immunother. 10, 607-614 (2014).

32. Henrickson, K. J. \& Hall, C. B. Diagnostic assays for respiratory syncytial virus disease. Pediatr. Infect. Dis. J. 26, S36-S40 (2007).

33. Nokes, D. J. et al. Incidence and severity of respiratory syncytial virus pneumonia in rural Kenyan children identified through hospital surveillance. Clin. Infect. Dis. 49, 1341-1349 (2009). 